\title{
Inhaltsübersicht
}

Seite

Vorwort .......................

Inhaltsverzeichnis $\ldots \ldots \ldots \ldots \ldots \ldots \ldots \ldots \ldots \ldots$ IX

Systematisches Musterverzeichnis. . . . . . . . . . . . XV

Abkürzungsverzeichnis .................. XXI

Literaturverzeichnis . . . . . . . . . . . . . . . . . XXVII

\section{A. Einführung}

Rn. Seite

I. Aufgaben, Personal und Organisation der

Staatsanwaltschaft $\ldots \ldots \ldots \ldots \ldots \ldots \ldots \ldots \ldots \ldots$

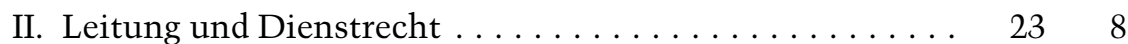

\section{B. Arbeitsplatz und -technik des Staatsanwalts}

I. Die Akten . . . . . . . . . . . . . . . . . . . . . . . . . 51

II. Aktenbearbeitung und Verfügungstechnik . . . . . . . $80 \quad 25$

III. Berichtswesen . . . . . . . . . . . . . . . . . . . . . . 11536

IV. Hilfsmittel zur rationellen Arbeit . . . . . . . . . . . . . . . . . 12540

V. Umgang mit Publikum und Medien . . . . . . . . . . . . . 14245

\section{Ermittlungsverfahren}

I. Zuständigkeit des Staatsanwalts . . . . . . . . . . . . . . . 14947

II. Einleitung des Verfahrens . . . . . . . . . . . . . . . . . $165 \quad 53$

III. Ziel und Gegenstand des Ermittlungsverfahrens . . . . . . . 22170

IV. Die am Ermittlungsverfahren Beteiligten . . . . . . . . . 25178

V. Einzelne Ermittlungshandlungen . . . . . . . . . . . . . 379116

VI. Fahndung und Haft . . . . . . . . . . . . . . . . . . . . . . 537167

\section{Entscheidung über Einstellung oder Anklage}

I. Verfahrenseinstellung gemäß $§ 170$ II StPO . . . . . . . . . . 600187

II. Einstellungen aus Opportunitätsgründen . . . . . . . . . . . 652207

III. Erhebung der öffentlichen Klage . . . . . . . . . . . . . . . 749242

IV. Besondere Verfahrensarten . . . . . . . . . . . . . . 867287 


\section{E. Das Verfahren zwischen Anklage und Urteil}

I. Zwischenverfahren . . . . . . . . . . . . . . . . . 877293

II. Hauptverhandlung . . . . . . . . . . . . . . . . . . . . . . . . . . . 889295

\section{F. Die Rechtsmittel}

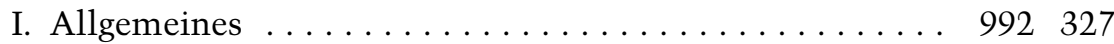

II. Beschwerde . . . . . . . . . . . . . . . . . . . . . . . 999328

III. Berufung . . . . . . . . . . . . . . . . . . . . . . . . 1015334

IV. Revision . . . . . . . . . . . . . . . . . . . . . . 1030340

V. Rechtsbeschwerde in Ordnungswidrigkeitenverfahren . . 1054349

VI. Wiederaufnahme des Verfahrens . . . . . . . . . . . . . . . 1056350

\section{G. Vollstreckungsverfahren}

I. Maßnahmen nach Beendigung des Hauptverfahrens . . . . . 1065353

II. Strafvollstreckung . . . . . . . . . . . . . . . . . . . . . . . . 1084359

III. Gnadenwesen . . . . . . . . . . . . . . . . . . . . . . . . . . . . . 1182390

Stichwortverzeichnis . . . . . . . . . . . . . . . . . . . . . . 397 


\section{Inhaltsverzeichnis}

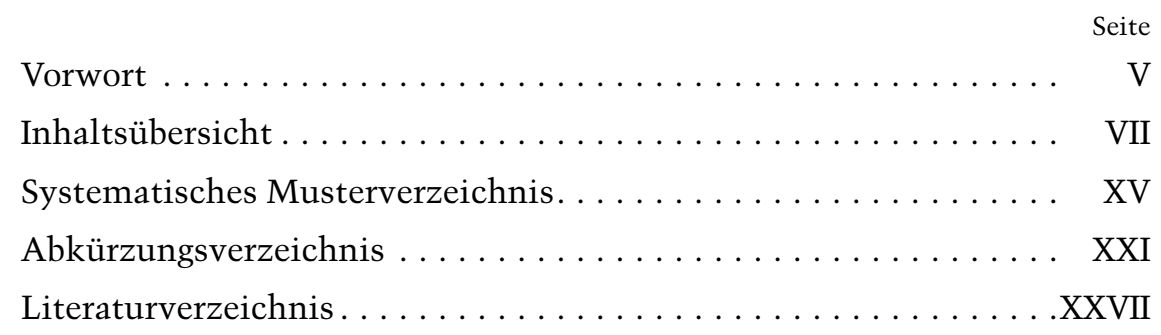

\section{A. Einführung}

Rn. Seite

I. Aufgaben, Personal und Organisation der Staatsanwaltschaft .................... 1

1. Die Staatsanwaltschaft als Organ der Rechtspflege . . . . . 111

2. Behördenstruktur ................. 73

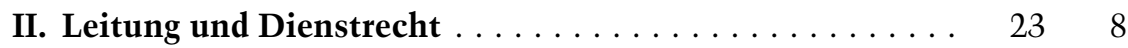

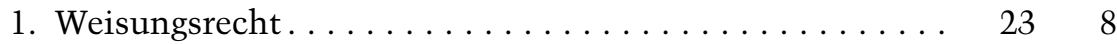

2. Zeichnungsbefugnis und Vortragspflichten . . . . . . . $30 \quad 10$

3. Ausschließung und Befangenheit des Staatsanwalts . . . . 4012

4. Haftung ...................... 4514

\section{B. Arbeitsplatz und -technik des Staatsanwalts}

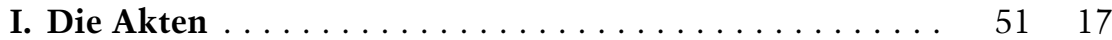

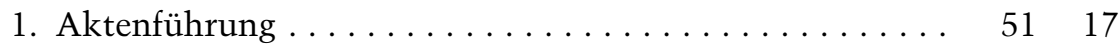

2. Besondere Akten . . . . . . . . . . . . . . . . . . . . . . 5418

3. Registrierung und Aktenzeichen . . . . . . . . . . . . . 5919

4. Verbleib der Akten nach Verfahrensende.......... 7423

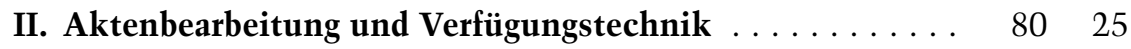

III. Berichtswesen . . . . . . . . . . . . . . . . . . . . . . $115 \quad 36$

IV. Hilfsmittel zur rationellen Arbeit . . . . . . . . . . . . . $125 \quad 40$

1. Organisation der Arbeit . . . . . . . . . . . . . . . . 12540

2. Abfassen von Verfügungen und anderen

Schriftstücken....................... 13142

3. Computer und Internet . . . . . . . . . . . . . . . . 13643

V. Umgang mit Publikum und Medien . . . . . . . . . . 14245 


\section{Ermittlungsverfahren}

Rn. Seite

I. Zuständigkeit des Staatsanwalts _. . . . . . . . . . . . . . . . $149 \quad 47$

II. Einleitung des Verfahrens . . . . . . . . . . . . . . . . . . . . . . 16553

1. Anfangsverdacht .......................... 16854

a) Exterritorialität . . . . . . . . . . . . . . . . . . . . . $178 \quad 57$

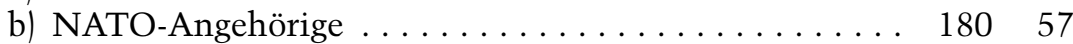

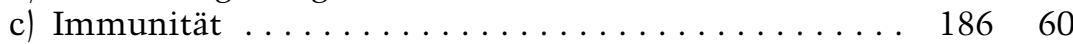

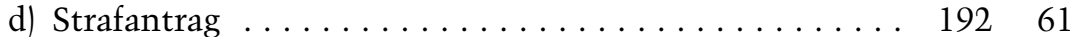

e) Veriährung . . . . . . . . . . . . . . . . . . . . . . . 19963

2. Verfahrenseinleitung. . . . . . . . . . . . . . . . . 20564

III. Ziel und Gegenstand des Ermittlungsverfahrens . . . . . . . 22170

1. Hinreichender Tatverdacht . . . . . . . . . . . . . . . 22371

2. Gegenstand der Ermittlungen . . . . . . . . . . . . . 24276

IV. Die am Ermittlungsverfahren Beteiligten . . . . . . . . . 25178

1. Staatsanwaltschaft ...................... 25178

a) Ermittlungsherrschaft .................... 25178

b) Akteneinsicht und Auskünfte . . . . . . . . . . . . . . $257 \quad 80$

2. Gericht .......................... $278 \quad 86$

3. Polizei und Ermittlungspersonen . . . . . . . . . . . . . 29693

4. Beschuldigter und Verteidiger . . . . . . . . . . . . . . . . . 319100

5. Der (mutmaßliche) Verletzte . . . . . . . . . . . . . . . . 363112

6. Sonstige Verfahrensbeteiligte ................. 374114

V. Einzelne Ermittlungshandlungen . . . . . . . . . . . . . 379116

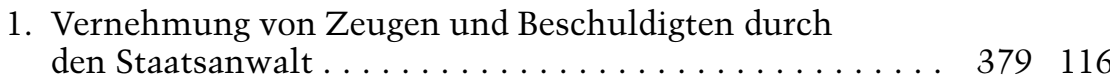

2. Auskunftsverlangen und Einsicht in fremde Akten ... . . 399122

3. Einholen von Gutachten . . . . . . . . . . . . . . . . . 414128

a) Auswahl von Sachverständigen. . . . . . . . . . . . . . . . 414128

b) DNA-Analysen . . . . . . . . . . . . . . . . . . . . . 4 424131

4. Beschlagnahme ...................... 434136

a) Sicherstellung zu Beweiszwecken . . . . . . . . . . . 435136

b) Sicherstellung nach $\S 111 \mathrm{~b}$ StPO . . . . . . . . . . $451 \quad 140$

c) Behandlung sichergestellter Sachen . . . . . . . . . $457 \quad 142$

d) Rückgabe sichergestellter Sachen . . . . . . . . . . . . . . . 465144

5. Herausgabeverlangen . . . . . . . . . . . . . . . . . 480149

6. Durchsuchung ......................... 484151

7. Ermittlungen in Datenverarbeitungsanlagen und -netzen . . . . . . . . . . . . . . . . . . . 502157

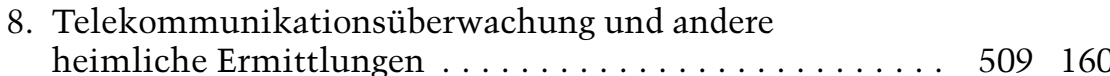

9. Leichenöffnung . . . . . . . . . . . . . . . . . . . . . 524163 
10. Verwertung erlangter Erkenntnisse zur Verfolgung anderer Delikte . . . . . . . . . . . . . . . . . . . . 533166

VI. Fahndung und Haft . . . . . . . . . . . . . . . . . . . . . . 537167

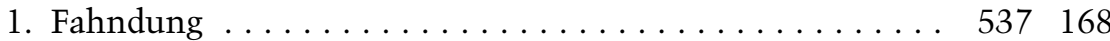

a) Lokale Fahndung . . . . . . . . . . . . . . . . . . . . . . . 539168

b) Bundesweite Fahndung . . . . . . . . . . . . . . . . . . . 541169

c) Öffentliche Fahndung . . . . . . . . . . . . . . . . . . 557173

d) Anordnungsbefugnisse . . . . . . . . . . . . . . . . . 560174

e) Fahndung im Ausland . . . . . . . . . . . . . . . . . 561175

2. Untersuchungshaft . . . . . . . . . . . . . . . . . . 569177

\section{Entscheidung über Einstellung oder Anklage}

I. Verfahrenseinstellung gemäß § 170 II StPO _ . . . . . . . 600187

1. Einstellungsverfügung ... . . . . . . . . . . . . . . . . . . 600187

a) Erteilung eines Einstellungsbescheides . . . . . . . . . . . . 604188

b) Einstellungsnachricht . . . . . . . . . . . . . . . . . . . . 616191

c) Teileinstellungen ......................... 621193

d) Behörden als Anzeigeerstatter . . . . . . . . . . . . . . . 627195

2. Inhalt des Einstellungsbescheides . . . . . . . . . . . . 632197

3. Rechtsmittel gegen die Einstellung . . . . . . . . . . . . . 640203

II. Einstellungen aus Opportunitätsgründen . . . . . . . . 652207

1. Einstellung gemäß $\S 153$ StPO . . . . . . . . . . . . . . 652207

2. Vorläufige Einstellung nach $\S 153$ a StPO gegen Auflagen .......................... 666211

3. Einstellung nach $\S 153 b$ StPO . . . . . . . . . . . . . . . . . 684218

4. Einstellung nach den $\S \S 153 \mathrm{c}, 153 \mathrm{f}$ StPO bei Auslandsbezug ..................... 686218

5. Vorläufige Einstellung nach $\S 154$ StPO . . . . . . . . . . 688219

6. Vorläufige Beschränkung der Verfolgung nach $\S 154$ a StPO . . . . . . . . . . . . . . . . . . . . 702224

7. Vorläufige Einstellung nach $\S 154 \mathrm{~b}$ StPO bei Auslieferung und Ausweisung .............. 708226

8. Einstellung nach $\S 154 c$ StPO gegenüber Erpressungs- und Nötigungsopfern . . . . . . . . . . . . . . 713227

9. Vorläufige Einstellung nach $\S 154 d$ StPO bei ziviloder verwaltungsrechtlichen Vorfragen . . . . . . . . . 715227

10. Vorläufige Einstellung nach $\S 154$ e StPO bei falscher Verdächtigung . . . . . . . . . . . . . . . 717228

11. Verweisung auf den Privatklageweg . . . . . . . . . . 721229 
12. Absehen von der Klage nach den $\S \S 31 a, 37$ BtMG . . . . . 724231

13. Einstellung bei Jugendlichen und Heranwachsenden nach $\S 45$ JGG . . . . . . . . . . . . . . . . . . . . 729232

14. Sonderfall des Täter-Opfer-Ausgleichs . . . . . . . . . 736236

15. Rechtsmittel gegen Einstellungen aus Opportunitätsgründen . . . . . . . . . . . . . . . . . 748242

III. Erhebung der öffentlichen Klage . . . . . . . . . . . . . . . 749242

1. Auswahl der Verfahrensart . . . . . . . . . . . . . . . . . 749242

2. Anklageschrift . . . . . . . . . . . . . . . . . . 759245

a) Zuständiges Gericht . . . . . . . . . . . . . . . . . . 760246

b) Angaben zur Person des Angeschuldigten . . . . . . . . 775250

c) Anklagesatz ...................... 781252

d) Beweismittel . . . . . . . . . . . . . . . . . . . . 816265

e) Ermittlungsergebnis . . . . . . . . . . . . . . . . . . . 824268

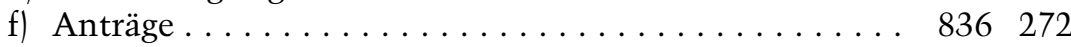

g) Anklageformular und -beispiel . . . . . . . . . . . . 839272

h) Abschluss- oder Begleitverfügung zur Anklage . . . . . . 841277

3. Antragsschrift im beschleunigten Verfahren . . . . . . . . 852281

4. Antragsschrift im vereinfachten Jugendverfahren . . . . . . 855283

5. Strafbefehlsantrag . . . . . . . . . . . . . . . . . . . 856283

IV. Besondere Verfahrensarten . . . . . . . . . . . . . . . . 867287

1. Antragsschrift im Sicherungsverfahren . . . . . . . . . 867287

2. Antragsschrift im objektiven Verfahren ... . . . . . . . 873289

\section{E. Das Verfahren zwischen Anklage und Urteil}

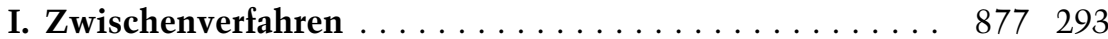

II. Hauptverhandlung . . . . . . . . . . . . . . . . . . . . . . . . . . 889295

1. Allgemeines ... . . . . . . . . . . . . . . . . . . 889296

2. Vorbereitung der Sitzung . . . . . . . . . . . . . . . . . . 893296

3. Beginn der Hauptverhandlung . . . . . . . . . . . . . . . . . . 899298

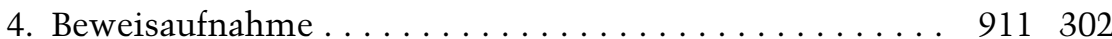

5. Öffentlichkeit und Störungen der Sitzung . . . . . . . . . . . 931308

6. Nachtragsanklage . . . . . . . . . . . . . . . . . . . . . 935308

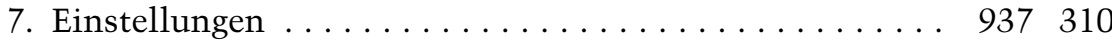

8. Verständigung über das Verfahrensergebnis . . . . . . . . . . 939310

9. Plädoyer ............................. 948313

a) Aufbau, Inhalt und Vortragsstil . . . . . . . . . . . . . . . 948313

b) Strafzumessungserwägungen ................. 956315

c) Vorbereiten und Halten des Plädoyers . . . . . . . . . . . . 971319 
10. Maßnahmen nach Urteilsverkündung . . . . . . . . . . . . 980323

11. Besonderheiten im Jugendverfahren . . . . . . . . . . . . . . 986325

\section{F. Die Rechtsmittel}

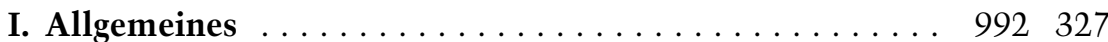

II. Beschwerde . . . . . . . . . . . . . . . . . . . . . . . . . . . 999328

1. Beschwerde der Staatsanwaltschaft . . . . . . . . . . . . 999328

2. Beschwerde des Beschuldigten .............. 1012333

III. Berufung . . . . . . . . . . . . . . . . . . . . . . . . 1015334

1. Berufung der Staatsanwaltschaft . . . . . . . . . . . . . . 1015334

2. Berufung des Angeklagten . . . . . . . . . . . . . . 1023338

IV. Revision . . . . . . . . . . . . . . . . . . . . 1030340

1. Revision der Staatsanwaltschaft . . . . . . . . . . . . . . . 1030340

2. Revision des Angeklagten . . . . . . . . . . . . . . . . . . . . . 1043344

V. Rechtsbeschwerde in Ordnungswidrigkeitenverfahren . . 1054349

VI. Wiederaufnahme des Verfahrens . . . . . . . . . . . . . . . . 1056350

1. Wiederaufnahme zu Gunsten des Verurteilten ........ 1056350

2. Wiederaufnahme zu Ungunsten des Beschuldigten . . . . . 1063351

\section{G. Vollstreckungsverfahren}

I. Maßnahmen nach Beendigung des Hauptverfahrens . . . . . 1065353

1. Allgemeines ........................ 1065353

2. Durchführung eines Entschädigungsverfahrens ...... 1075355

3. Nachträgliche Gesamtstrafenbildung . . . . . . . . . . . . . 1079357

II. Strafvollstreckung . . . . . . . . . . . . . . . . . . . . . . . . . 1084359

1. Allgemeines . . . . . . . . . . . . . . . . . . . . . . . . 1084359

2. Vollstreckung von Geldstrafen . . . . . . . . . . . . . 1093361

3. Vollstreckung von Freiheitsstrafen . . . . . . . . . . . . . . 1095361

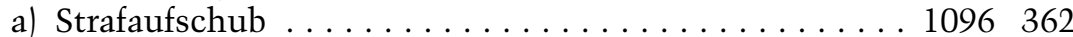

b) Zurückstellung der Strafvollstreckung nach $\S 35$ BtMG . . . . . . . . . . . . . . . . . 1105365

c) Absehen von der Vollstreckung bei Ausländern . . . . . 1114369

d) Strafunterbrechung und Hafturlaub . . . . . . . . . . . . 1126373

e) Aussetzung des Strafrestes . . . . . . . . . . . . . . . . 1131374 
Rn. Seite

f) Maßnahmen bei Vollverbüßung . . . . . . . . . . . . . . 1143377

g) Nachträgliche Sicherungsverwahrung . . . . . . . . . 1146377

4. Bewährungsstrafen . . . . . . . . . . . . . . . . 1155383

5. Vollstreckung von freiheitsentziehenden Maßregeln der Besserung und Sicherung . . . . . . . . . . . . . . . . . . 1174388

III. Gnadenwesen . . . . . . . . . . . . . . . . . . . . . . . . . . . 1182390

Stichwortverzeichnis . . . . . . . . . . . . . . . . . . . . . . . . . . . . 397 